\title{
"STEPPING INTO THE EQUATORWARD BOUNDARY OF THE AURORAL OVAL: PRELIMINARY RESULTS OF MULTI SCALE STATISTICAL ANALYSIS,
}

\author{
Massimo Materassi ${ }^{*}, 1$, Mirko Piersanti ${ }^{2}$, Giuseppe Consolini ${ }^{3}$, Piero Diego ${ }^{3}$, \\ Giulia D’Angelo ${ }^{4}$, lgor Bertello ${ }^{3}$, Antonio Cicone ${ }^{5}$ \\ (1) Istituto dei Sistemi Complessi, Consiglio Nazionale delle Ricerche, Firenze, Italy \\ (2) Istituto Nazionale di Fisica Nucleare - Sezione di Roma "Tor Vergata", Rome, Italy \\ (3) Istituto di Astrofisica e Planetologia Spaziali, Rome, Italy \\ (4) Dipartimento di Matematica e Fisica, Università degli Studi "Roma Tre”, Rome, Italy \\ (5) DISIM, Università degli Studi dell'Aquila, L'Aquila, Italy
}

Article history

Receveid June 4, 2018; accepted January 29, 2019.

Subject classification:

Space Weather; Plasma physics; Dynamics; Statistical analysis; Data processing.

\begin{abstract}
This work aims at showing how analysis techniques, designed to highlight the multi-scale structure of a signal, may be of help in describing fluctuations of the ionospheric medium. In particular, the technique used here is the ALIF (Adaptive Local Iterative Filter). We have applied ALIF to the characterisation of plasma irregularities in the equatorward boundary of the auroral oval. The data used are from the LEO satellite DEMETER (Detection of Electro-Magnetic Emissions Transmitted from Earthquake Regions) while it crosses the Equatorward boundary of the oval. The time series analysed are those from the electric field instrument ICE (Instrument de Champ Electrique) on board of DEMETER, and the identification of the equatorward boundary in the time series of in situ data is based on the comparison between the satellite trajectory and the picture of the auroral oval from the simultaneous auroral imaging by the DMSP satellite. In detail, we present an analysis of the multi-scale features and dynamics of ionospheric plasma along the DEMETER trajectory in different regions, which seem to be characterized by plasma irregularities of different nature. A clear change of the multi-scale nature of plasma fluctuations is observed in the correspondence of the irregularities originated by particle precipitation in the equatorward boundary of the auroral oval.
\end{abstract}

\section{INTRODUCTION}

Auroral ovals (A0) are the regions located in the northern and southern high latitude ionosphere where the main particles coming from magnetosphere precipitate [Feldstein, 1963; Feldstein et al., 1969; Akasofu, 1966]. These particles interact with the atmospheric atoms and molecules giving rise to luminosity in both visible and ultraviolet spectra. At ionospheric altitudes, the aurorae appear as continuous and luminous bands surrounding both geomagnetic poles [Frey, 2007].

Moving from the large number of observations and models, Feldstein [1963] and Khorosheva [1967] suggested the concept of "auroral oval", that high-latitude region between $65^{\circ} \mathrm{N}(\mathrm{S})$ and $78^{\circ} \mathrm{N}(\mathrm{S})$ where aurorae are generally observed. Essentially, the AO consists in two parts: the discrete oval and the diffuse oval. The last one lies mainly equatorward of the discrete oval, while diffuse precipitation, although often not visible, appears also poleward of the discrete oval. The discrete arcs are more dy- 
namic in space (size, shape and location) and time (from second to minutes) than the diffuse oval [Borovsky, 1993]. Naturally, the dynamics and the position of the AOs strictly depend on the geomagnetic activity level. In fact, its equatorward boundary (EWB) location depends on both the energy of the precipitating particles and on the magnetospheric electric and magnetic fields [Kauristie et al., 1999; Villante and Piersanti, 2010; Piersanti and Villante, 2016; Piersanti et al., 2017]. Notwithstanding, its poleward boundary is defined as the separation between the closed field lines and the polar cap.

The boundaries of the AO can be determined either by ground-based observations [Moen et al., 2004; Aikio et al., 2006] and by low earth orbit (LEO) satellite observations [Wang et al., 2005]. In addition, global view snapshots of the AO can be provided through optical images taken from high-altitude satellites [Østgaard et al., 2007]. On this topic, using the DMSP (flowing at around $850 \mathrm{~km}$ at sunsychronous orbit) data, Newell et al. [2009] modelled the monoenergetic discrete aurora and the AO boundaries.

Closely related to the AO location, a current system, which is directly driven by the interaction between the solar wind and the plasma magnetically bounded to the Earth, continuously flows along geomagnetic field lines into and out of the ionosphere (field aligned currents FACs). Iijima and Potemra [1978] found that FAC sheets are aligned with the boundary of the AO during quiet geomagnetic conditions, while distortions of this alignment occur during disturbed periods. Xiong et al. [2014a] developed an efficient algorithm to detect the auroral boundaries from FAC signatures by using 10 years observations of the magnetic field from CHAMP (CHAllenging Minisatellite Payload). In a successive statistical analysis, based upon the same CHAMP dataset, Xiong et al. [2014b] developed an empirical model able to efficiently determine both the equatorward and the poleward boundaries of the AO.

Given its high variability and richness on many space and time scales, the AO can be conceived as a space- and time-multiscale complex dynamical structure. Still, it is well localised conceptually in terms of geomagnetic line morphology: in fact, its EWB is where the geomagnetic lines change between dipolar lines and those stretching several Earth radii into the geomagnetic tail of the Earth. The AO poleward boundary (PWB), instead, is where the geomagnetic lines stop to be closed (relatively near the Earth), and open deeply in the near-Earth space and solar wind, reconnecting with the interplanetary magnetic field.

Crossing the EWB, as one moves poleward from the region of dipolar geomagnetic field lines to that where geomagnetic lines stretch into the Earth's magnetic tail, the local physical quantities $\Phi(x, t)$ describing the ionospheric plasma (e.g., the electron and ion densities $n_{e}(x, t), n_{i}(x, t)$, or their local temperatures $T_{e}(x, t)$ and $\left.T_{i}(x, t)\right)$ will change. Specifically, the quantities $\Phi(x, t)$ pass from the values characteristic of the plasma populating the low and mid latitude ionosphere (or plasmasphere, depending on the height of the trajectory one describes (Vellante et al., 2014a,b)), to those pertaining to the plasma coming from the magnetic tail. Moreover, in the correspondence of the EWB, the static plasma of the dipolar region gives place to plasma fluxes arriving from the tail, so a strong shear in plasma velocity is expected, possibly producing instabilities, and hence strong fluctuations. Thus, across the EWB, local plasma quantities $\Phi$ will change not only in their "background" value $\Phi_{0}$ but also in their "fluctuating" part $\delta \Phi$, so that one has $\Phi=\Phi_{0}+\delta \Phi$. The local proxy will change in terms of fluctuation-to-background ratio $\delta \Phi / \Phi_{0}$ and, possibly, of the statistical distribution of these $\delta \Phi$ values.

Plasma boundary layers, as the EWB, are characterised by a major relevance of irregularities (i.e., the ratio $\delta \Phi / \Phi_{0}$ ) because turbulent regions are met: one often refers to turbulent boundary layers, indeed. Boundary layers are very interesting "mixed" regions. In fact, because of the high gradients to which the plasma undergoes, conditions of strong non-linear coupling between many degrees of freedom hold. This makes the plasma motion definitely nonlaminar and instabilities give rise to irregularities at many different time and space scales. As the dynamical features of different time and space scales (e.g., particle and kinetic effects, turbulent structures, continuum excitations) interact with each other, single approach treatments may result unsatisfactory: this is the real complexity of such an environment. The characterization of plasma proxies $\Phi=\Phi_{0}+\delta \Phi$ with fluctuations behaving differently at different scales, i.e. a multi-scale statistical analysis (MSSA) of the $\delta \Phi$ local fields, may open new perspectives in the comprehension of this complex dynamics.

In the present paper, we study the behaviour of different scale components of a suitable plasma proxy measured along the trajectory of a LEO satellite, as specified below. As part of the selected proxy time series $\Phi(t)$ of the in situ measurements lays within the EWB of the AO, and part outside of it, our approach allows for a comparison between the two plasma regions in terms of MSSA. In detail, we examine some intervals of in situ data relative to the floating potential $V_{\text {float }}$ collected by the ICE (Instrument Champ Electrique, Berthelier et al., (2006)) experiment onboard of the DEMETER (Detection of Electro-Magnetic Emissions Transmitted from Earthquake Regions) satellite [Lagoutte et al., 2006], when the 
satellite was entering the northern $\mathrm{AO}$ crossing the EWB plasma. This physical quantity (i.e., our $\left.\Phi(t)=V_{\text {float }}(t)\right)$ is an indirect proxy of the local plasma features and dynamical state. The floating potential $V_{\text {float }}(t)$, even if not trivially related to the local plasma quantities (more directly to $n_{\mathrm{e}}$ and $T_{\mathrm{e}}$ ), is expected to be rather faithful to them, both in terms of its trend behaviour and fluctuations. Moreover, the high rate sampling of $V_{\text {float }}(t)$ allows to study the tiny details of the irregularities met. This grants to highlight how the passage between the topsideplasmaspheric environment and the EWB plasma is neither a sudden jump nor a smooth transition, but rather a midland in which it is possible to meet, sporadically but clearly, plasma features and structures characterizing both the two regions. If the use of a pictorial image is allowed, this is roughly what the driver meets when the car starts to enter a rainy region, and sees the first droplets on the windshield before the pouring rain envelops the car (of course, in the case of a satellite entering the EWB, the droplets are the first irregular localized plasma structures of the EWB). As already anticipated, the proxy $V_{\text {float }}(t)$ is multi-scale analysed via the ALIF technique [Piersanti et al., 2018].

We remark that the analysis in this work is not intended to diagnose the entrance of the satellite into the EWB, but rather to introduce a tool to compare the multi-scale statistical dynamics of the plasma in different points of the trajectory that are presumed to be located in different plasma regions. In fact, the identification of the EWB in the $V_{\text {float }}(t)$ time series is based on the comparison between the satellite trajectory and the picture of the AO from the simultaneous auroral imaging of the DMSP satellite, see Section 3.

The paper is organized as follows.

In Section 2 the data analysed and the method used are presented. The quantities calculated for the analysis proposed are illustrated in some detail. Section 3 thoroughly describes the 4 case events at hand, giving detailed explanations of the general appearance of the time series $V_{\text {float }}(t)$ analysed, and sketching the near-Earth space context from which it comes. In Section 4, the results of our analysis are presented and duly commented; then, some conclusions are drawn and future research directions sketched.

\section{DATA AND METHODS}

In this Section we describe the dataset used in our analysis, which are relative to the DEMETER mission, launched on June 29, 2004 and devoted to study the Earth's ionosphere and the disturbances due to seismic activities. Let's shortly remind that the DEMETER satellite orbits on a sun-synchronous orbit, located at 10:30 LT (local time) and at 22:30 LT, with an inclination of about $98.23^{\circ}$ and an altitude of about $700 \mathrm{~km}$. The DEMETER satellite performs 14 orbits per day and measures continuously between $-65^{\circ}$ and $+65^{\circ}$ of geographical latitude, and its payload includes, among others, electric field sensors (ICE), magnetic sensors (IMSC), and Langmuir probes (ISL). Here, we make use of the ICE experiment data.

\subsection{ICE DEMETER MEASUREMENTS}

In order to investigate the high latitude ionospheric fluctuation features we analyse data from the electric potential of the ICE (Instrument Champ Electrique) probe on DEMETER while crossing the northern high latitude EWB layer [Berthelier et al., 2006; Badoni et al., 2018]. In addition, the correspondent Langmuir probe measurements are used to detect plasma parameters (ion $n_{\mathrm{i}}$ and electron $n_{\mathrm{e}}$ density, and electron temperature $T_{\mathrm{e}}$ ) which at the DEMETER orbit according to the international reference ionosphere (IRI) model should range as follows: plasma density $n_{\mathrm{i}}=n_{\mathrm{e}}$ between $5 \cdot 10^{9}$ and $5 \cdot 10^{11} \mathrm{~m}^{-3}$; electron temperature $T_{\mathrm{e}}$ between $2000 \mathrm{~K}$ and $3500 \mathrm{~K}$.

ICE probes acquire the floating potentials $\left(V_{\text {float }}\right)$ in ULF band at a frequency of $39 \mathrm{~Hz}$ [Berthellier et al., 2006]. In general, the floating potential follows the fluctuations of plasma potential $\left(V_{\mathrm{pl}}\right)$. In the case of the ICE probes, four contributions are relevant: electron collection, ion collection, photoelectron emission, and the current injected to the probe by the embedded current bias source. The behaviour of $V_{\text {float }}$ is modelled by the rates of particles collections as described by the equations reported in Diego et al. (2017).

In an ideal case, plasma density variations do not affect $V_{\text {float }}$ since ion and electron collections have equal and opposite dependence from $n$, and bias and photoelectron contributions could be considered almost negligible at locations along the DEMETER orbit. However, in case of strong plasma density reductions, such contributions could be of the same order of electron current and, therefore, more effective in moving positive the $V_{\text {float }}$.

DEMETER ICE probes have been developed for an accurate (of the order of $\mu \mathrm{Vs}$ ) measurement of the floating potential with the aim to detect electric fields in the range DC-4 MHz. Sensors are designed to operate at plasma densities greater than $10^{10} \mathrm{~m}^{-3}$. For this reason, ICE time series are very suitable for the study of rapid changes in ionospheric environment, such as the discontinuities of auroral zone. On the other hand, Langmuir probe measurements remain of good reliability but are not adequate for the monitoring of such small structure in the high latitude plasma. 


\subsection{DATA SEGMENTS ANALYSED}

The four case events considered are the ones reported in Table 1 below, namely: January 1, 2005; January 1, 2008; June 1, 2010; July 2, 2010.

\begin{tabular}{lccc} 
Case Event & UT & AE Index & Dst Index \\
January 1, 2005 & $6.3<$ UT $<6.48$ & $500 \mathrm{nT}$ & $-13 \mathrm{nT}$ \\
January 1, 2008 & $14.5<\mathrm{UT}<14.7$ & $125 \mathrm{nT}$ & $-17 \mathrm{nT}$ \\
June 1, 2010 & $6.7<\mathrm{UT}<7.0$ & $310 \mathrm{nT}$ & $-30 \mathrm{nT}$ \\
July 2, 2010 & $14.2<\mathrm{UT}<14.5$ & $425 \mathrm{nT}$ & $-16 \mathrm{nT}$ \\
\hline
\end{tabular}

TABLE 1. A table resuming the helio-geophysical proxies of the case events analysed.

We choose this data segments, because of their almost homogeneous geophysical conditions (in terms of $\mathrm{AE}$ and Dst indices), ensuring DEMETER satellite passing through the $\mathrm{AO}$.

\subsection{MULTISCALE STATISTICAL ANALYSIS (MSSA)}

In many dissipative dynamical systems outside of equilibrium, the observed fluctuations are very large and result from the interaction of a high number of degrees of freedom. When such an interaction yields multi-scale and chaotic spatio-temporal field fluctuations, and involve a nonlinear transfer of energy among the different spatio-temporal scales (e.g., via a direct and/or inverse cascading mechanism as for instance the Richardson's cascade), the system is generally said to display turbulent dynamics. More in general, quoting Falkovich [2008], a turbulent state can be defined "as a state of a physical system with many interacting degrees of freedom deviated far from equilibrium. This state is irregular both in time and in space and is accompanied by dissipation". The present analysis of the time series of the ionospheric proxy $V_{\text {float }}(t)$ is inspired by the tools used in the study of turbulent media, which manifest large amplitude multiscale fluctuations and high spatial gradients due to the couplings of their components [Frisch, 1995].

The study of signals collected probing turbulent media often deals with highly erratic time series $X(t)=X_{0}(t)+\delta X(t)$, and hence must be treated statistically (in the foregoing expression, $X_{0}(t)$ is the "low pass component" of $X(t)$, while fluctuations $\delta X(t)$ are generally considered to be the "high pass component"). The statistical analysis are well based for data intervals within which the statistics of the signal fluctuations $\delta X(t)$ is stationary. Hence, the first step to apply this reasoning to the study of our fluctuating time series $V_{\text {float }}(t)$ is to select sub-intervals of the whole time series within which fluctuations $\delta V_{\text {float }}(t)$ have a stationary statistics.

As a suitable interval $I$ for our analysis is fixed, the statistics of the fluctuations $\delta X(t)$ are studied at different time resolutions, in order to single out those different scale-fixed degrees of freedom interacting non-linearly in the complex medium. In particular, the differences $\delta_{\tau} X(t)=\delta X(t+\tau)-\delta X(t)$ are studied separately for each $\tau$, so to obtain the probability distribution functions $P_{I}\left(\delta_{\tau} X\right)$ for different values of $\tau$. The dependence on $\tau$ of the statistics of the values $\delta_{\tau} X(t)$ will give information on the physical mechanisms governing the fluctuations $\delta X(t)$. In the following, we will refer to this study of the dependence of $P_{I}\left(\delta_{\tau} X\right)$ on $\tau$ as multi-scale statistical analysis (MSSA) of fluctuations.

As a first step, the MSSA requires a multi-scale decomposition of the signal $\delta X(t)$, giving rise to the series $\delta_{\tau} X(t)$. This decomposition may either be done through the simple definition $\delta_{\tau} X(t)=\delta X(t+\tau)-\delta X(t)$, or via some more formal decomposition of $\delta X(t)$ into "independent" components $\delta_{\tau} X(t)$ with characteristic variability length $\left\langle\frac{\delta_{\tau} \dot{X}}{\delta_{\tau} X}\right\rangle \approx \tau$, being $\langle\psi\rangle$ the time average of the quantity $\psi(t)$ over the time interval of stationary statistics for $\psi$. Such decomposition into scale-fixed independent modes

$$
\delta X(t)=\sum_{\tau} \delta_{\tau} X(t)
$$

is generally obtained as

$$
\delta_{\tau} X(t)=\sum_{t_{0}} g\left(\tau, t_{0}\right) w_{\tau, t_{0}}(t)
$$

the collection of functions $w_{\tau, t_{0}}$ is a set of independent modes, each of has $\left\langle\frac{w_{t, 0}}{\dot{w}_{t, 0}}\right\rangle \approx \tau$, with support centred in $t_{0}$. The coefficients $g\left(\tau, t_{0}\right)$ give the relevance of the -sized mode in a neighbourhood of $t_{0}$ to the composition of $\delta X(t)$. Without loss of generality, the expression (1) may be constructed with orthonormal $w_{\tau, t_{0}}$, so that:

$X(t)=\sum_{t_{0}} g\left(\tau, t_{0}\right) w_{\tau, t_{0}}(t), \int_{t_{i}}^{t_{f}} w_{\tau, t_{0}}^{*}(t) w_{\tau^{\prime}, t_{0}^{\prime}}(t) d t=\delta_{t_{0}, t_{0}^{\prime}{ }_{0}} \delta_{\tau, \tau^{\prime}}(2)$

(the deltas in (2) are Kronecker symbols, being the set $\left\{w_{\tau, t_{0}}\right\}$ necessarily countable in data analysis). The MSSA consists then in the characterization of how the distribution of those values depends on $\tau$, i.e. how $P_{I}\left(\delta_{\tau} X\right)$ changes with $\tau$. The functions $w_{\tau, t_{0}}(t)$ in (1) may be of various nature, basically equivalent. Adaptive Local Iterative Filtering (ALIF), where $w_{\tau, t_{0}}(t)$ is a set of signal-adapted functions, was used in Piersanti et al. [2018] to obtain multi-scale filters as (2) for GPS radio scintillation signals. In that work the authors put in evidence 
how MSSA from discrete wavelets and ALIF give roughly the same diagnosis for the turbulent signal examined. In the present paper, the ALIF technique has been adopted to construct all the -dependent statistical functions (hereafter called intrinsic mode components - IMCs). We have however to remark that using the above definition of $\delta_{\tau} X(t)$ is not exactly the same of that used in the classical approaches to the study of turbulence media (Frisch, 1995). Indeed, here we do not deal with increments at a fixed scale $\tau$ but, rather, with the fluctuation contribution at a certain characteristic scale $\tau$. This approach is quite different and it has been already applied in the framework of turbulent space plasma studies via similar decomposition techniques [see, e.g., Consolini et al., 2017].

The distributions $P_{I}\left(\delta_{\tau} X\right)$ can be characterized via some quantities (cumulants) describing their shape: each statistical parameter obtained from $P_{I}\left(\delta_{\tau} X\right)$ will depend on the scale $\tau$ at hand (and on the interval). In order to check the $\tau$-dependence of $P_{I}\left(\delta_{\tau} X\right)$, its first statistical momenta are usually considered: in particular, the second cumulant and the fourth normalized one: the variance $\sigma_{I}^{2}(\tau)$ and the kurtosis $K_{I}(\tau)$. The normalized kurtosis, or better the difference $K_{I}^{e x}(\tau)=K_{I}(\tau)-3$, indicated as excess of kurtosis, represents how the distribution tails differs from those of the Gaussian distribution.

It may also make sense to calculate the Shannon entropy

$$
I_{I}(\tau)=-\sum_{\delta_{\tau^{X}}} P_{I}\left(\delta_{\tau} X\right) \log _{2} P_{I}\left(\delta_{\tau} X\right)
$$

which can provide a measure of the "degree of randomness" of the fluctuations $\delta_{\tau} X$. Furthermore, in order to highlight how the $\tau$-th component $\delta_{\tau} X$ stands out at a particular time $t$ in terms of "energy", the ratio $\varepsilon_{\text {rel }}(t, \tau)$ between the quantity $\left|\delta_{\tau} X(t)\right|^{2}$ and its integral over the scales is calculated:

$$
\varepsilon_{\text {rel }}(t, \tau)=\frac{\left|\delta_{\tau} X(t)\right|^{2}}{\int d \tau^{\prime}\left|\delta_{\tau} X(t)\right|^{2}}
$$

The quantity, named local intermittency measure (LIM) [Farge, et al., 1990], is widely use in turbulent studies to identify the signal structures which are responsible for the occurrence of intermittency. In order to stress even more the (time) intermittency of the series $\delta X(t)$, the square of LIM is calculated, and simply named $\mathrm{LIM}^{2}$ :

$$
\operatorname{LIM}^{2}(t, \tau)=\frac{\left|\delta_{\tau} X(t)\right|^{4}}{\left\langle\left|\delta_{\tau} X(t)\right|^{2}\right\rangle_{t}^{2}}
$$

The two quantities, $\varepsilon_{\text {rel }}(t, \tau)$ and $\mathrm{LIM}^{2}$, allow for locating the sites where the fluctuation squared amplitude (energy) exceeds the average one or the intermittent events $\left(\mathrm{LIM}^{2}>3\right)$. Thus, while the variance, kurtosis and Shannon information of $P_{I}\left(\delta_{\tau} X\right)$ are related to global features of the signal in the interval $I$ as seen at the scale $\tau$, the LIM $\varepsilon_{\text {rel }}(t, \tau)$ in (4) and the $\mathrm{LIM}^{2}$ in (5) rather define local properties of the $\delta_{\tau} X$ at time $t$.

In what follows, the MSSA is applied to the time series $V_{\text {float }}(t)$ collected by the ICE instrument on board of DEMETER. In detail, we show a collection of results from the different tools mentioned above. Furthermore, each time series $V_{\text {float }}(t)$ is first subdivided into two intervals: the "pre" interval, pertaining to the plasma within the dipolar geomagnetic field; the "post" interval, where the satellite crosses tailward stretched geomagnetic lines. Two sub-series $V_{\text {float }}^{\text {pre }}(t)$ and $V_{\text {float }}^{\text {post }}(t)$ are selected accordingly, and the MSSA difference between their features are highlighted.

\section{RESULTS OF THE ANALYSIS}

In this Section we present in detail the results about multi-scale analysis of the case events mentioned in Session 2 .

\subsection{THE JANUARY 1, 2005 CASE EVENT}

Figure 1 shows the ICE and ISL observation during the DEMETER orbit segment between 5.88 UT and 6.48 UT. The offset observed between $N_{i}$ and $N_{e}$ is an instrumental effect. In fact, Langmuir probes (Lp), performs the various plasma particle collections at different polarization level (i.e. current collected along a sweeping voltage applied) to obtain the so called I-V characteristic from which it is possible to derive plasma parameters (Chen, 1977). Since the relevant currents values are known to have different slope, the computational uncertainty of $V_{\mathrm{pl}}$ detection gives rise to a computational difference between $N_{i}$ and $N_{e}$.

In general, it is expected that $V_{\text {float }}(t)$ (panel 3) follows the theoretical floating potential $\left(V_{\mathrm{f}, \mathrm{th}}\right.$, panel 4), obtained through the plasma data measured by $L p$ as reported in Diego et al. (2017). For the event under investigation it does not happened. In fact, while before 6:43 UT (vertical purple dashed line), $V_{\text {float }}(t)$ is characterized by an almost monotonichal increase and presents an unexpected growth at about 6.2 UT, $V_{\mathrm{f}, \mathrm{th}}$ shows an almost constant value $(\approx 1 \mathrm{~V})$. This inconsistency is related to the concurring contribution of highly plasma variations (see the large enhancement of about $3 \mathrm{~V}$, occurring between 6.2 UT and $6.43 \mathrm{UT}$ ), that changes the particles collection mode of the probe, and to the motion of the satellite across 


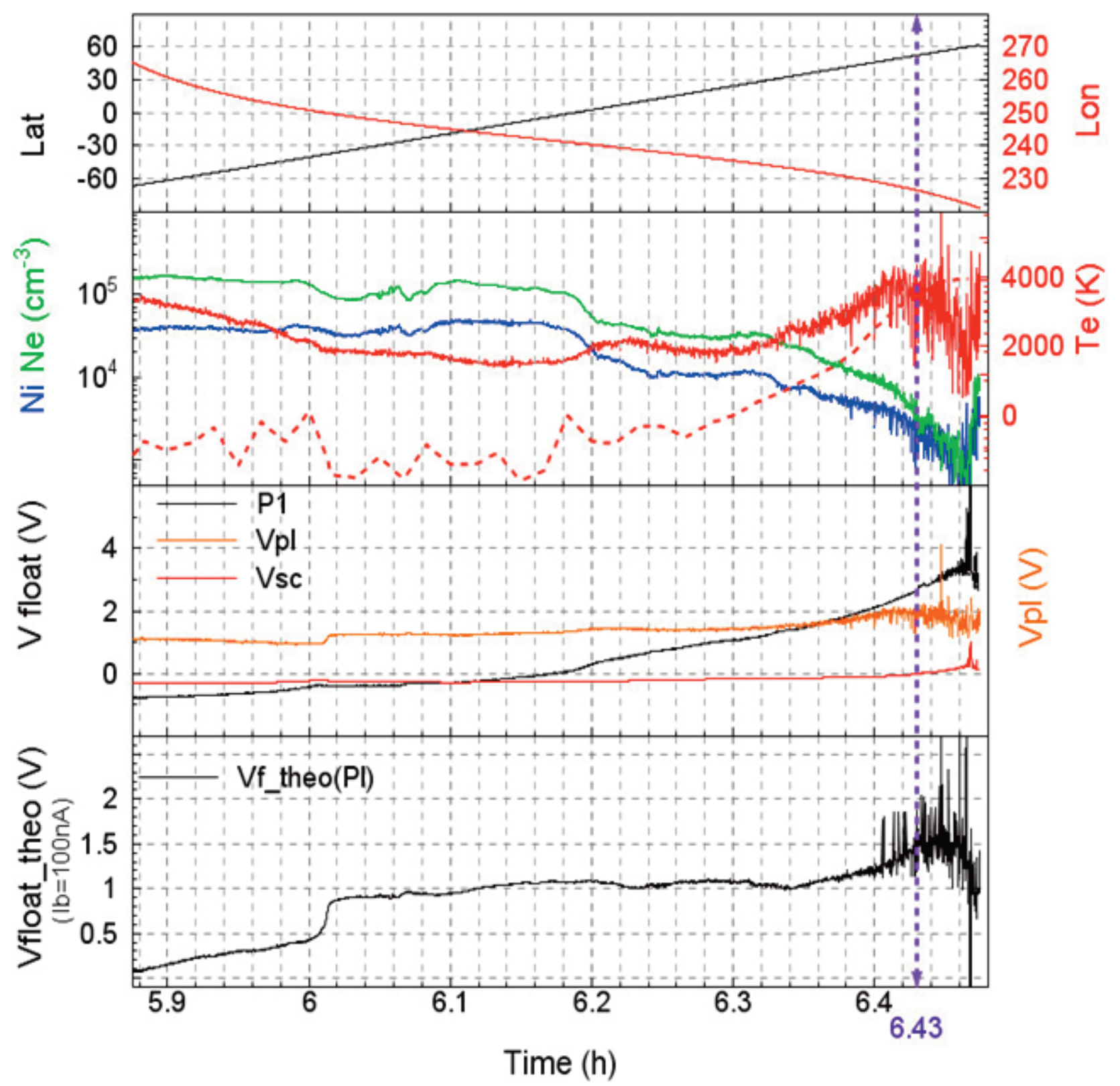

FIGURE 1. DEMETER ICE and ISL data relevant to the 5th orbit of January 1th, 2005. Top panel shows satellite latitude and longitude, second panel display the ion and electron density (blue and green curve respectively), the electron temperature and its standard deviation (red curve and red dotted curve respectively) computed every minute. Third panel shows one ICE probe floating potential (black), along with plasma potential (orange) and S/C potential (red). Bottom panel shows the theoretical curves obtained with each current computed with the correspondent density ( $I_{e}$ computed by using $N_{e}$, and $I_{i}$ by using $N_{i}$ ). Time is reported in decimal units UT. The vertical purple dashed line represents the time of 6.43 UT.

an increasing geomagnetic field. The first effect increases the ratio $\lambda_{\mathrm{D}} / \mathrm{R}_{\text {probe }}$, invalidating the above mentioned thick sheath approximation. The second effect reduces the electron gyroradius, causing a no more isotropic collection of electrons by the probe. The consequence is a positive growth of $V_{\text {float }}(t)$. Interestingly, between 6.3 UT and 6.43 UT, the plasma densities (panel 2, blue and green lines) and the electron temperature (panel 2 red line) show a sharp decrease and a sharp increase, respectively. This behaviour is explained by the crossing of DEMETER satellite through the "ionospheric trough" [Hunsucker and Hargreaves, 2007]. Finally, the sharp decrease of plasma density appearing at 6.2 UT, is clearly a seasonal effect caused by the equator crossing of the satellite, passing from summer to winter [Whitteker et al., 1978; Sojka, 1982].

The same but more marked situation appears in $V_{\text {float }}(t)$ 
January 1, 2005 DOY:001 Orbit: 06223(DMSPF16)
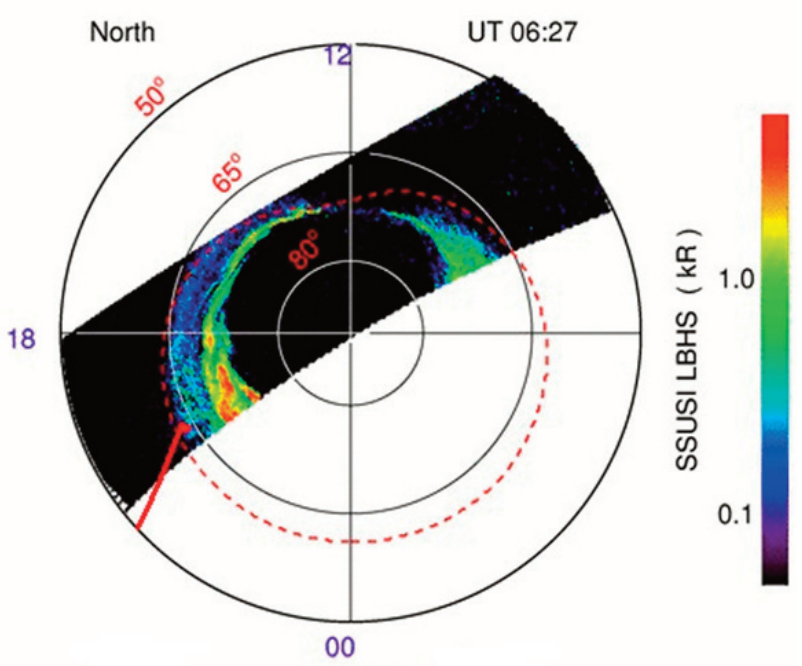

FIGURE 2. The trace of DEMETER's orbit (red) drawn on the top of the Auroral Oval map on January 1, 2005. The arc of orbit spans from UT 06:23:56 to UT 06:28:29, and it is superimposed on the map of Auroral Oval emission (in kilo-Röntgen) taken at UT 06:27.

after 6.43 UT. In fact, it is first characterized by fluctuations whose amplitudes increases with increasing latitude, and then, it shows huge and sudden variations. At the same time, the relative plasma parameters show a strong decrase of the electron temperature, coupled with a decrease in the plasma density. $T_{e}$ depends on the transition region slope as well as the probe-plasma impedance. Thus, since the current of particle collection directly depends on their number, it is normal to observe enhanced and noisy temperature values in an extraordinary lowdensity plasma. On the other hand, in such condition the computational differences in $N_{e}$ and $N_{i}$ tend to disappear as can be seen in Figure 1 (third panel), since the electron and ion currents slopes become similar, and the uncertainties of $\Delta \mathrm{I} / \Delta \mathrm{V}$ ratio near the inflection point $\left(V_{\mathrm{pl}}\right)$ are similar as well.

The peculiar behaviour of $V_{\text {float }}, n_{e}$ and $T_{e}$ after 6.43 UT can be related to the entrance of the satellite into a ionospheric boundary. The geographical position of the satellite between 6.43 UT and 6.48 UT renders it sensible to expect the satellite to be entering the EWB of the $\mathrm{A} 0$, as confirmed by Figure 2: there, the superposition of the DEMETER orbit (red line) on the A0 observations detected by DMSP satellite is reported.

The selected orbit arc (Figure 2) refers to the last 2 minutes (about $800 \mathrm{~km}$ ) of the analysed data segment, precisely the interval corresponding to the irregularity in the Lp data. Comparing the trace of the red arc in Figure 2 to the time series of $V_{\text {float }}(t)$ reported in Figure 1, it is visible that the occurrence of the peaks of $V_{\text {float }}^{\text {post }}(t)$ takes place exactly at the latitudes of the outermost auroral oval. A point has to be clarified in this respect. The DEMETER satellite flies at $700 \mathrm{~km}$, that is well above the region from which auroral emission comes. That emission is produced by the particle precipitation along the geomagnetic field lines: we believe that the irregular structure met in the time series of data from its Lp $V_{\text {float }}(t)$ corresponds to the plasma irregularities upstream the emission origin region, caused by the same particle precipitation switching on the aurora below.

On on the basis of the $V_{\text {float }}(t)$ behavior, we divided our observations into two time series: the first one $\left(V_{\text {float }}^{\text {pre }}(t)\right)$, which starts at 6.3 UT and terminates at 6.43 UT (outside the $\mathrm{A} 0)$; the second one $\left(V_{\text {float }}^{\text {post }}(t)\right)$, which begins at 6.43 UT and finishes at 6.48 UT (inside the A0).

\subsection{MSSA RESULTS}

To analyze the multi-scale statistics of the in situ irregularities of the medium corresponding to the pouring particle precipitation determining the Auroral Oval, $V_{\text {float }}(t)$ between 6.3 UT and 6.48 UT is considered, concentrating, however, on $V_{\text {float }}^{\text {post }}(t)$ (Figure 3). As the time series $V_{\text {float }}(t)$ is reported in panel (a) of Figure 3, its subseries $V_{\text {float }}^{\text {pre }}(t)$ and $V_{\text {float }}^{\text {post }}(t)$ are singled out by glance. When entering the EWB the $V_{\text {float }}$ becomes more spiky, as it is clear from the middle panel (b) where $V_{\text {float }}^{\text {post }}(t)$ is report$\mathrm{ed}$, and the difference between adjacent elements of time, $\delta V_{\text {float }}^{\text {post }}(t)$, reported in the bottom panel (c) (that is useful to highlight apparent intermittency).

Time-scale analysis is possible for all these time series, which turns into being a space-scale analysis because of the satellite-plasma relative motion. It is worth specifying that a trend has been subtracted from the time series of Figure 3 in order to highlight the fluctuations, whose analysis represents the core of this paper. In addition, we can neglect the baseline behaviour in because all its physical proprieties are already identifiable in its detrended version.

The plot of the time series $V_{\text {float }}(t)$ in Figure 3 (panel a) indicates at a glance how an irregular structure follows the smoother part of the series. A first simple but effective way of comparing the two data segments $V_{\text {float }}^{\text {pre }}(t)$ and $V_{\text {float }}^{\text {post }}(t)$ is to calculate their power spectral density (PSD) via Fourier analysis showed in the panels (a) and (b) of Figure 4, respectively. The spectrum of $V_{\text {float }}^{\text {pre }}(t)$ in Figure $4 \mathrm{a}$ is characterised by a strong peak at $1 \mathrm{~Hz}$ on the top of a smooth power spectrum background representing rather weak noise; the $1 \mathrm{~Hz}$ peak is due to an experimental artefact of the Langmuir probe (Lebreton et al. 2006; Logoutte et al., 2005). 


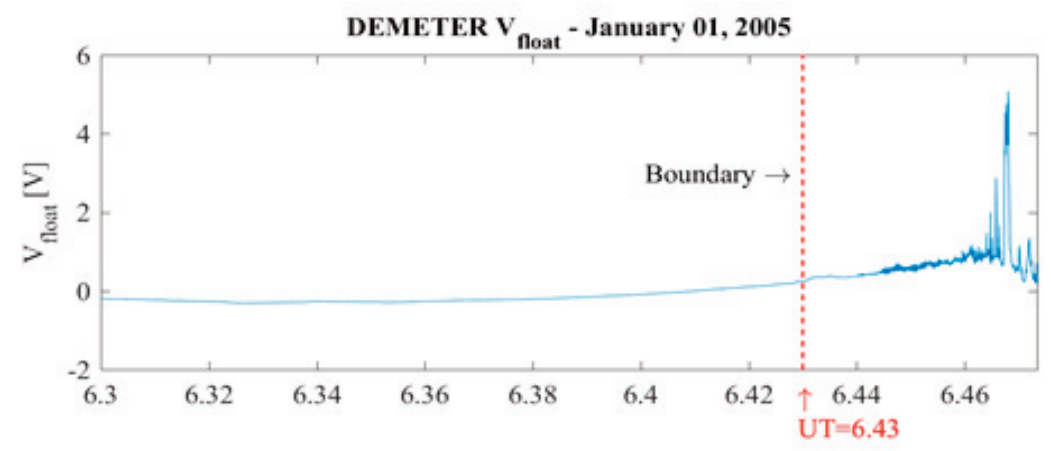

a)

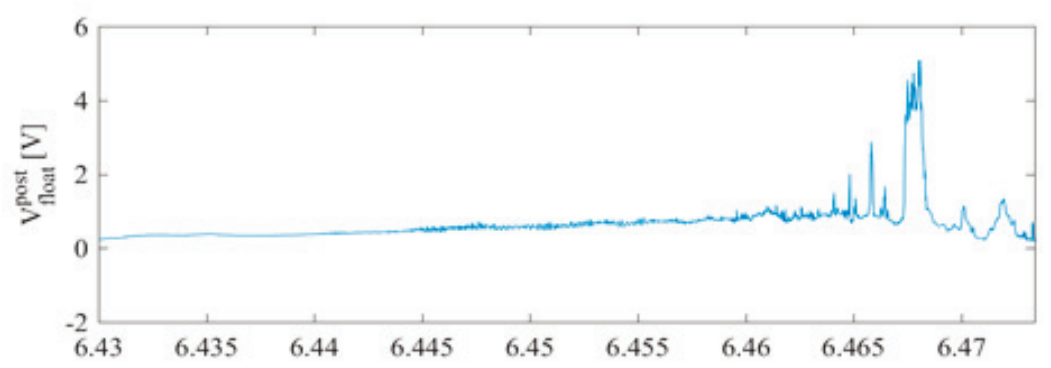

b)

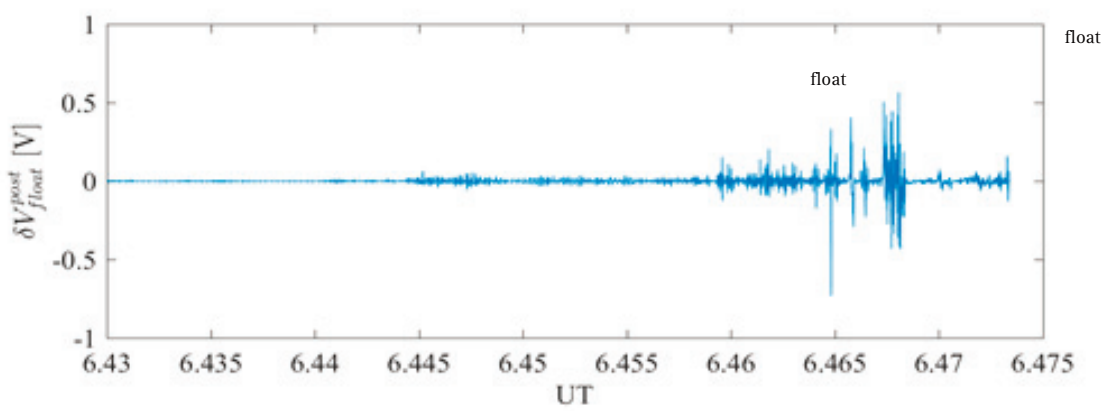

c)

FIGURE 3. From top to bottom: the whole time series (panel a) of the floating potential, the sub-series $V_{\text {float }}^{\text {post }}(t)$ (panel b) collected inside the EWB, and the difference between adjacent elements of time, $\delta V_{\text {float }}^{\text {post }}(t)$ (panel c).

The Figure $4 \mathrm{~b}$ plot represents instead the PSD of $V_{\text {float }}^{\text {post }}(t)$ : the time series collected in the EWB plasma is a double power-law spectrum with two distinct regimes, i.e., a lower frequency power law with exponent $\alpha_{1}=-1.48 \pm 0.42$, and a higher frequency power law with exponent around $\alpha_{1}=-2.69 \pm 0.22$. This kind of behaviour, definitely pointing towards multi-scale dynamics, is not easily recognizable in terms of well-known and accepted theories. This is a preliminary indication that in the EWB it is very likely that some plasma instability may generate a multi-scale power-law spectrum. Furthermore, the power-law character of PSD suggests that scale invariance may be a peculiar feature of such fluctuations. However, this point requires more analysis that are outside the scope of this work and are demanded to a future more detailed study (see Section 4 for more comments).

When $V_{\text {float }}(t)$ is ALIF-decomposed, a change of regime is observed at $t^{*}=6.43 \mathrm{UT}$, becoming apparent as a mul- ti-scale element. Figure 4c and Figure 4d, report the relative energy $\varepsilon_{\text {rel }}(f, t)$ for the whole time series $V_{\text {float }}(t)$ and $\operatorname{LIM}^{2}(f, t)$, as functions of the equivalent instantaneous frequency $f$ (assuming $f=\tau^{-1}$, see Piersanti et al. (2018)) and time $t$, respectively. The $\varepsilon_{\text {rel }}(f, t)$ behaviour (Figure 4c) shows that the energy distribution is definitely different before and after $t^{*}$. Before $t^{*}$ the whole segment is characterized by the strong $f=1 \mathrm{~Hz}$ component, while after $t^{*}$ a strong $f=2 \mathrm{~Hz}$ component appears. Looking at Figure $4 \mathrm{~d}$, reporting the $\operatorname{LIM}^{2}(f, t)$ of the $V_{\text {float }}^{\text {post }}(t)$ time series, at around $10 \mathrm{~Hz}$, smaller and sporadic plasma structure appear where the intermittency is highly concentrated.

The proper analysis of multi-scale statistics of $V_{\text {float }}(t)$ is performed by analyzing the two aforementioned segments $V_{\text {float }}^{\text {pre }}(t)$ and $V_{\text {float }}^{\text {post }}(t)$. Each segment is assumed to be statistically homogeneous (which is true in first approximation, and however their internal statistical variability is much less that the difference between the two ones), 

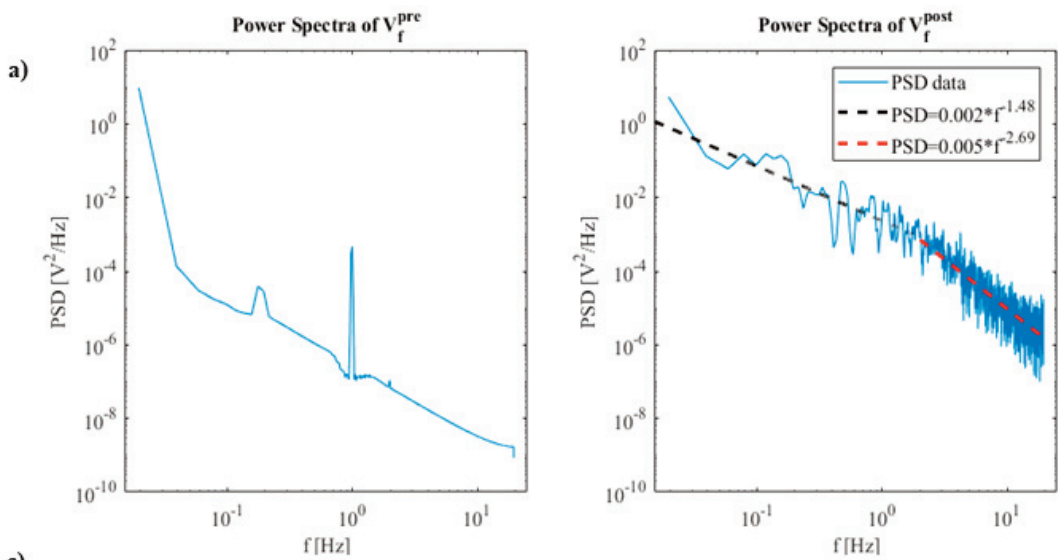

b)

c)
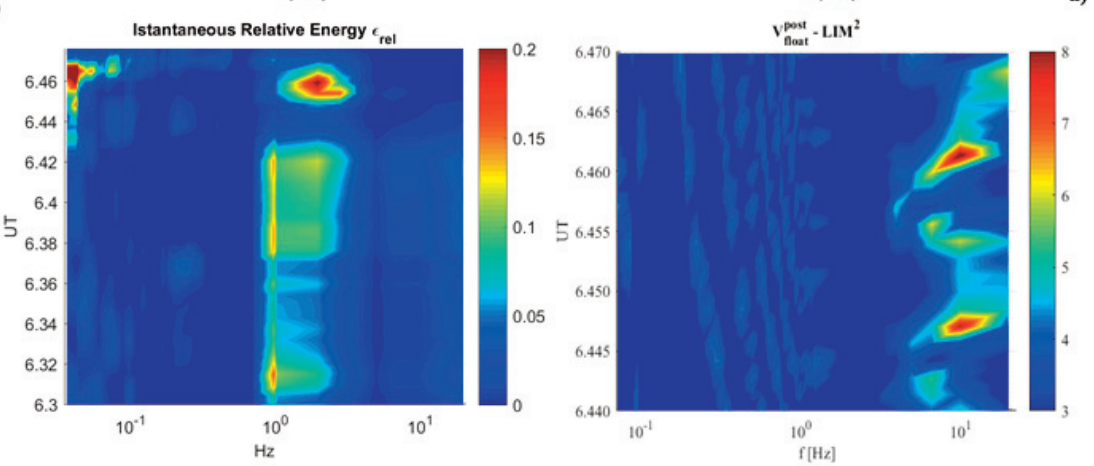

FIGURE 4. Upper panels: results of the spectral analysis of $V_{\text {float }}^{\text {post }}(t)$ (panel a) and $V_{\text {float }}^{\text {post }}(t)$ (panel b). The red and black dashed lines represent the best fit of the power spectrum. Lower panels: the result of the MSSA analysis on the whole time series $V_{\text {float }}^{\text {post }}(t)$, see the text

allowing its ALIF decomposition and MMSA analysis in a time-independent way. Then, the statistical parameters $\varepsilon_{\mathrm{rel}}(f), K(f)$ and $I(f)$ are calculated for each component, of equivalent Fourier frequency $f$, and reported as functions of $f$. The resulting plots for the smooth part of the $V_{\text {float }}^{\text {pre }}(t)$ series are reported in Figure 5, while, in Figure 6, the results for the irregular part of the series $V_{\text {float }}^{\text {post }}(t)$ are shown. Here, the time does not appear as a variable anymore, because the statistics of the whole (presumed homogeneous) time series $V_{\text {float }}^{\text {pre }}(t)$ and $V_{\text {float }}^{\text {post }}(t)$ have been considered together.

By comparing Figures 5 and Figure 6, three main features may be highlighted as important characteristics of the $V_{\text {float }}^{\text {pre }}(t)$-versus- $V_{\text {float }}^{\text {post }}(t)$ "transition". First of all, as already visible in Figure 4, the energy becomes differently distributed along the scales, so that smaller structures appear in the irregular portion of the crossed medium. Moreover, while in Figure 5 the function $K(f)$ is almost flat, with an increase between $3 \times 10^{-1} \mathrm{~Hz}$ and $1 \mathrm{~Hz}$, in Figure $6 K(f)$ is growing monotonically for frequencies higher than $1 \mathrm{~Hz}$, indicating that the smaller the structures is considered, the less-Gaussian distribution they show. In the presence of fully developed turbulence, which is not ascertained to be the case here, this would be safely stated as a condition of anomalous scaling, i.e., of in- termittency [Frisch, 1995].

Last but not least, in the comparison between the multi-scale statistics of the smooth and the irregular parts of the medium (Figures 5 and Figure 6, respectively), the $I(f)$ variability as a function of frequency $f$ has to be considered. An important feature is that the $K f)$ and $I(f)$ slopes seems to be "anti-correlated" in both cases. In addition, in both cases, $I(f)$ are different according to the difference between the two excesses of kurtosis. The "anti-correlation" of $I(f)$ with respect to $K(f)$ denotes that, as the $f$-fixed fluctuations depart from the Gaussian distribution and their kurtosis grows, they become less random showing a smaller Shannon entropy. This anticorrelation suggests that the shape of the PDFs of the fluctuations acquires a more leptokurtotic feature by decreasing the scale $\tau$ (as shown in Figure 7).

Last focus on the irregular behaviour of plasma at the EWQ of the Auroral Oval is reported in Figure 7, where the PDFs $P\left(x_{\tau}\right)$ distributions is reported for the various $\tau$ scales. Here, to compare the shape of the PDFs at different scales, the fluctuations $\delta_{\tau} \mathrm{X}(t)$ have been scaled to unit variance, i.e., $x_{\tau}=\frac{\delta_{\tau} X}{\sigma}$ where $\sigma$ is the standard deviation. These PDFs are clearly leptokurtotic showing fat tails and a very pronounced probability peak around zero in the core of the distribution. 

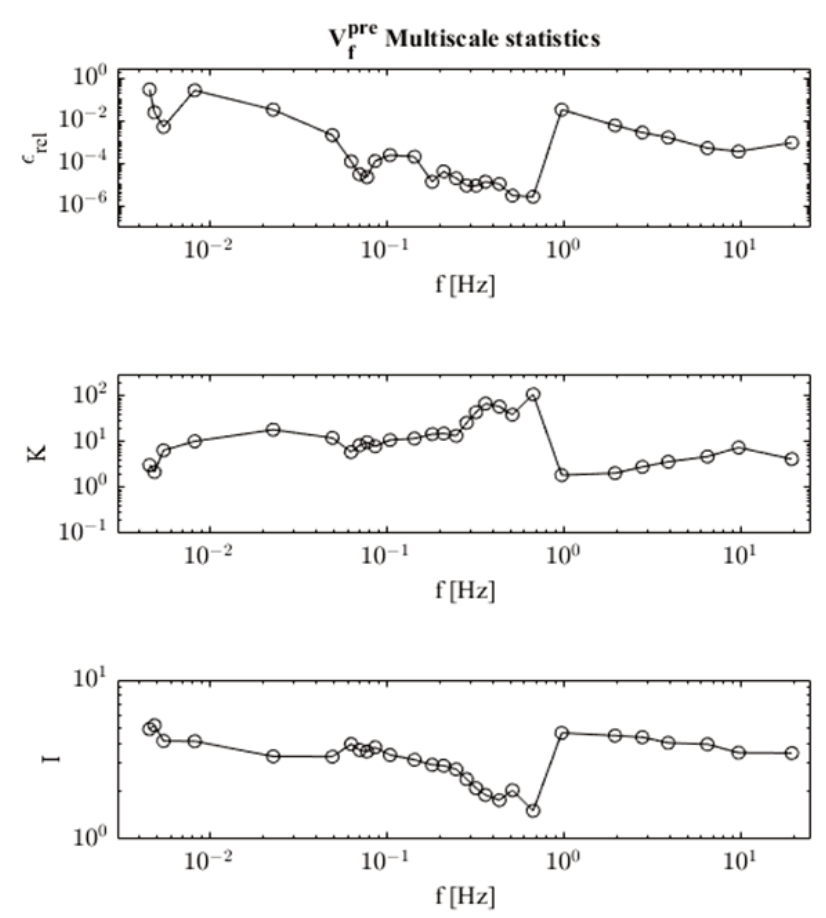

FIGURE 5. The result of the ALIF analysis on the pre-boundary time series $V_{\text {float }}^{\text {pre }}(t)$. From top to the bottom: the relative energy $\varepsilon_{\text {rel }}(f)$, the kurtosis $K(f)$ and the Shannon entropy $I(f)$ of the ALIF components as functions of the equivalent frequeny $f$.

The semilog plot clearly indicates that the PDFs do not collapse on a unique master shape. This behaviour indicates that the statistics of fluctuations of $V_{\text {float }}^{\text {post }}$ is not merely scale invariant, but conversely there is a change of the PDF shape from long to short timescales. In turbulent media, this is one of the signatures of intermittency, i.e. the crowd of localized features shows a scale dependence that is point dependent, and gives rise to multifractal features. This is another indication of the highly irregular and complex dynamics weaving the small scale texture of the EWB.

\subsection{THE OTHER EVENTS (JANUARY 1, 2008; JUNE 1, 2010; JULY 2, 2010).}

Figure 8 shows, on the left, the trace of DEMETER's orbit (red) drawn on the top of the Auroral Oval map on January 1, 2008 (top), June 1, 2010 (middle) and July 2, 2010 (bottom), as in Figure 2. On the right, figure 2 reports the PSD analysis for the corresponding events. As for the January 1, 2005 event, it can be seen that, as DEMETER flew in/out of the A0, the PSDs of each $V_{\text {float }}^{\text {post }}$ time series collected in the EWB plasma is a double powerlaw spectrum with two distinct regimes (a lower frequency power law and a higher frequency power law), suggesting again that scale invariance may be a peculiar feature of such fluctuations.

When each $V_{\text {float }}(t)$ is ALIF-decomposed, a peculiar $t^{*}$
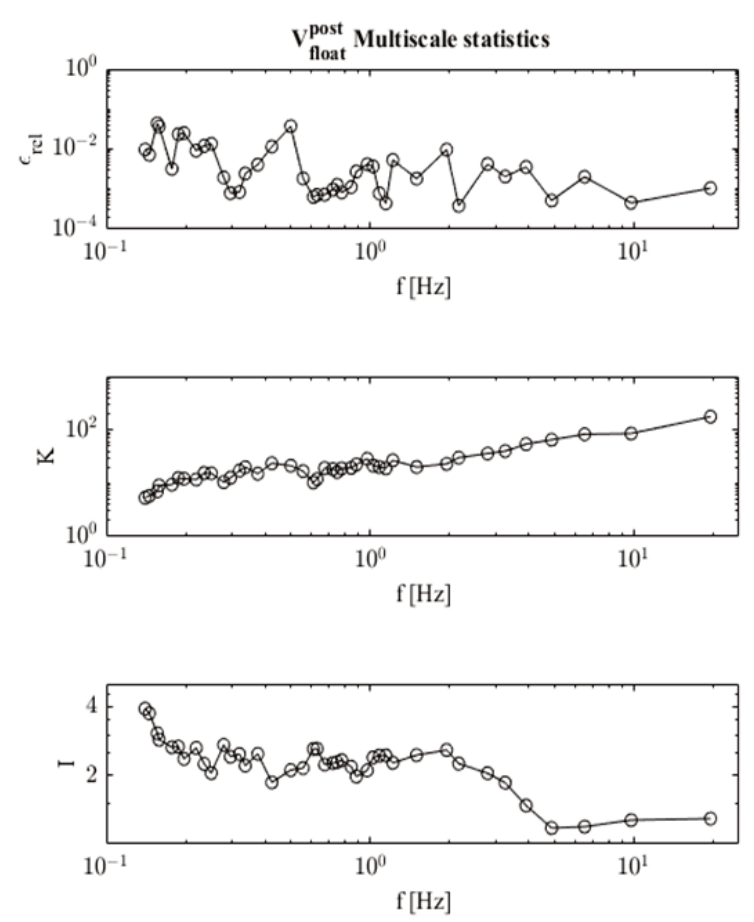

FIGURE 6. The result of the ALIF analysis on the post-boundary time series $V_{\mathrm{f}}^{\text {post }}(t)$. Form top to the bottom: the relative energy $\varepsilon_{\text {rel }}(f)$, the kurtosis $K(f)$ and the Shannon entropy $I(f)$ of the ALIF components as functions of the equivalent frequeny $f$.

appears, at which a change of regime is observed, becoming apparent as a multi-scale element. Figure 9 shows a summary plot of the MSSA applied to the July 2, 2010 event. Left panels show the statistical parameters $\varepsilon_{\text {rel }}(f)$, $K(f)$ and $I(f)$ calculated for each ALIF component of $V_{\text {float }}^{\text {post }}$ as functions of the frequency $f$. Right panels shows the $\mathrm{LIM}^{2}$ parameter (top) and the probability density function of $V_{\text {float }}^{\text {post }}$ for $1 \mathrm{~Hz}<\mathrm{f}<10 \mathrm{~Hz}$. The analysis confirms the results obtained for the January 1, 2005. In particular, the relative energy peaks at several different scales, indicating the presence of multi-scale structures, while the kurtosis grows up with $f$ indicating the same "intermittent appearance" seen in the $V_{\text {float }}^{\text {post }}$ segment studied in $\S \S 3.1$ and 3.2. In the same way, the fluctuations with higher kurtosis appear to be less Shannon-entropic, i.e. likely to be more deterministic, more "organized". Last but not least, the multi-scale PDF plot clearly indicates a non-collapse of the reduced distribution, probably indicating a not-self-similar behaviour of turbulent dissipation in the plasma in the EWB.

The for the other 3 case events, that may be consulted in the supplementary material of the paper, just confirm the same diagnosis about the EWB plasma studied via multi-scale statistics. 


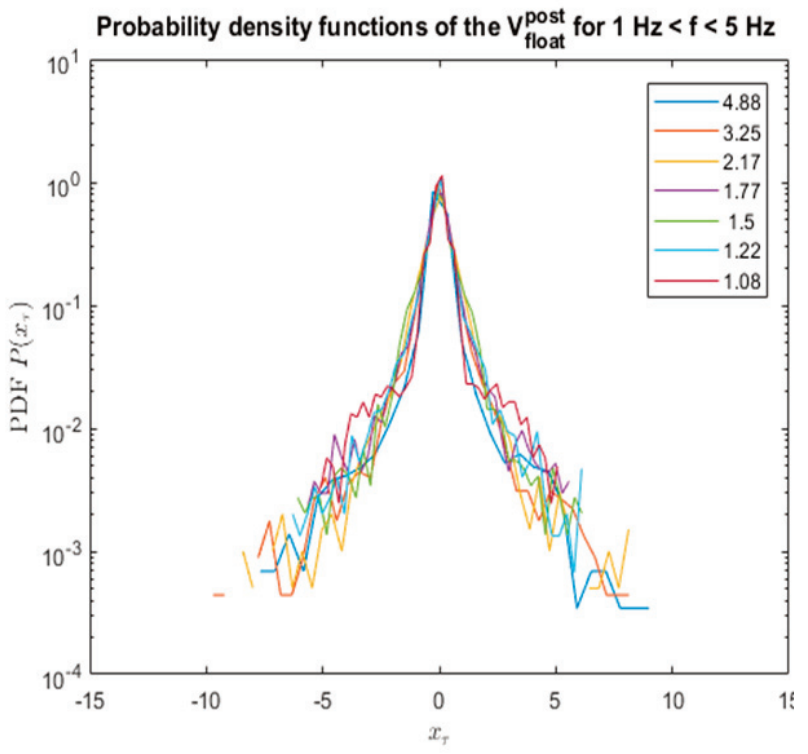

FIGURE 7. The standardized PDF for the fluctuations of $V_{\text {float }}^{\text {post }}(t)$ at frequencies between $1 \mathrm{~Hz}$ and $5 \mathrm{~Hz}$.

\section{CONCLUSIONS}

In this work we have presented the results of applying MSSA to the high latitude fluctuations observed by ICE experiment on-board of DEMETER, while crossing the EWB of the northern auroral oval. Indeed, thanks to the availability of the high rate $V_{\text {float }}(t)$ time series, one is able to perform a MSSA of the signatures of the ionospheric plasma irregularities on electric field measurements. In particular, the present paper addresses the MSSA of data collected in the outermost border of the A0, by applying the ALIF technique to multi-scale decompose the $V_{\text {float }}(t)$ time series, which is able to highlight the irregular structures sitting along the geomagnetic lines, approximately $700 \mathrm{~km}$ higher than the A0. The observed region should be that of the trails of particle precipitation giving rise to auroral emission.

Our analysis shows that fluctuations produced by the particles re-entering from the magnetotail along the geomagnetic lines are characterized by features pointing towards spatio-temporal intermittency. This statement is true under the reasonable hypothesis that the medium remains approximately steady while the probe crosses it (namely, the Taylor Hypothesis, see Frisch (1995)).

The take home message suggested by our results is the following.
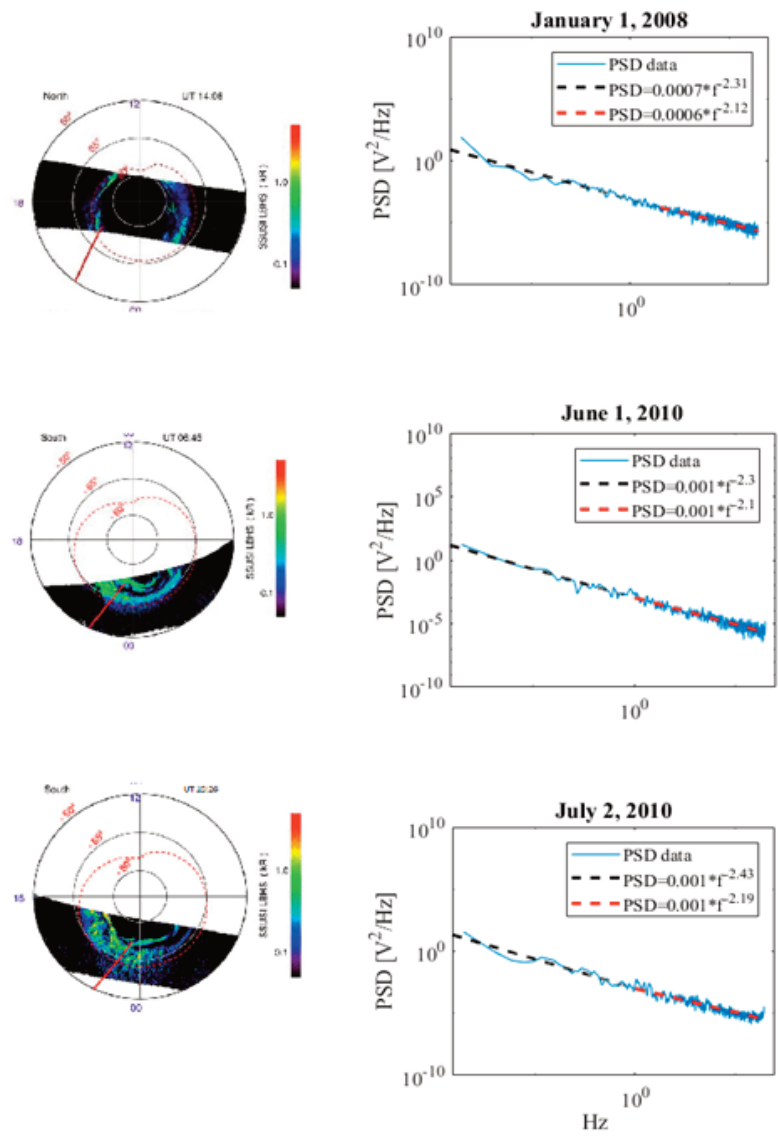

FIGURE 8. Left panels: the trace of DEMETER's orbit (red) drawn on the top of the Auroral Oval map on January 1, 2008 (top), June 1, 2010 (middle) and July 2, 2010 (bottom). The arc of orbit is superimposed on the map of Auroral Oval emission (in kilo-Röntgen). Right panels: the PSD of each "inside-theEWB" segment $V_{\text {flost }}^{\text {post }}$ always showing a double power-law regime (see the text).

The most external part of currents flowing in the auroral region is an intermittent distribution of irregular, localized structures. Their presence produces fluctuations in $V_{\text {float }}(t)$ that show higher and higher kurtosis distributions as smaller and smaller space details are examined (in Figure 6, indeed, $K(f)$ grows with $f$, as also for $K(f)$ in Figure 9, and in the respective plots in the supplementary material). This-suggests that, at the EWB of the A0, the ionospheric plasma medium can be in a turbulent state displaying patchy multi-scale structures. A further characterization of the small scale fluctuations of the plasma at the EWB is done by checking the shape of their reduced PDF at different scales (frequencies), seeing that, due to the fat tail behaviour, those probability distributions do not collapse. In practice, this means that fluctuations at different scales show more and more leptokurtotic statistics as smaller and smaller scales are considered. If one wanted to envis- 

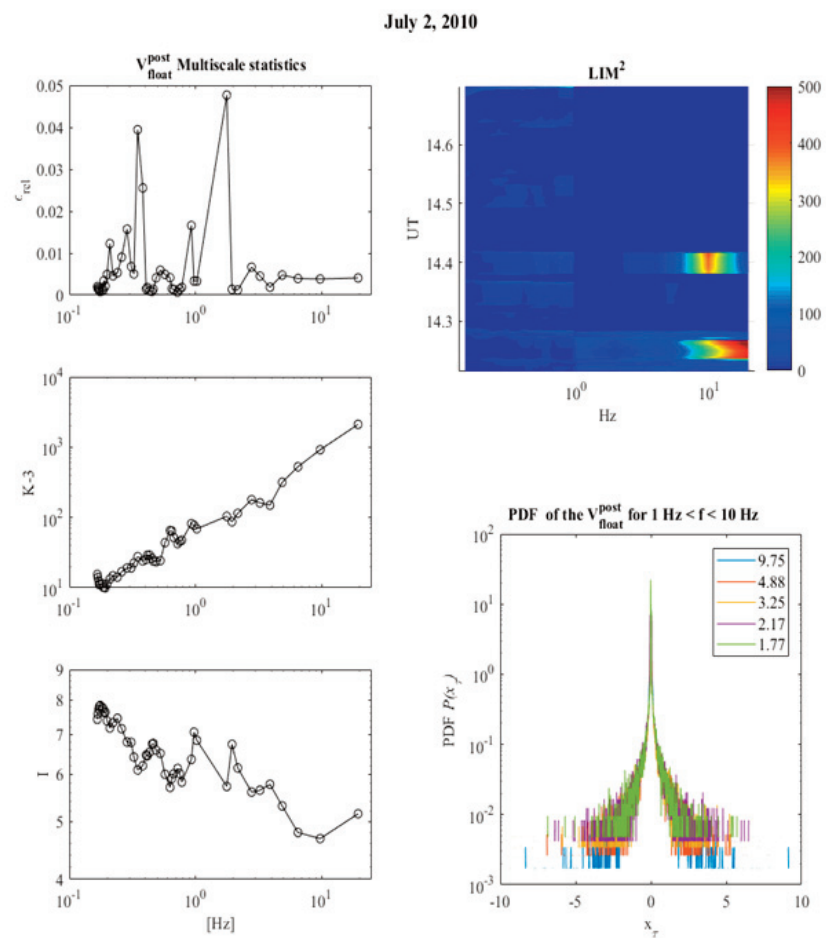

FIGURE 9. Left panels: the result of the ALIF analysis on the post-boundary time series $V_{\mathrm{f}}^{\text {post }}(t)$. Form top to the bottom: the relative energy $\varepsilon_{\text {rel }}(f)$, the kurtosis $K(f)$ and the Shannon entropy $I(f)$ of the ALIF components as functions of the equivalent frequeny $f$; right panels: $\mathrm{LIM}^{2}$ of $V_{\text {float }}^{\text {post }}$ (top) and the standardized PDF for the fluctuations of $V_{\text {float }}^{\text {post }}$ at frequencies between $1 \mathrm{~Hz}$ and $10 \mathrm{~Hz}$.

age a theoretical picture justifying such results, it would be necessary to state that from scale to scale the behaviour of dissipation field is not scale-invariant (nonself-similar, multifractal).

All in all, at the EWB the plasma shows a nearly turbulent state, the origin of which could be related to the growing of some type instabilities (e.g., Kelvin-Helmotz, Kruskal-Shafranov, etc) in the boundary regions where large shear velocity gradient could be present. In Figure 2 this irregular nature of the AO boundary structure seems to appear already in the distribution of emissions registered by DMSP. However, when the fluctuations of the plasma are directly monitored through the in situ proxy $V_{\text {float }}(t)$, this "irregular appearance" turns out to be the real texture of that ionospheric region. Remarkably, such irregular texture occurs where a "boundary" is well known to exist (in this case, the EWB of the A0), suggesting that ionospheric "boundaries" may appear much richer and more complex if studied in detail.

The results presented in this paper are just preliminary ones, due to the limited amount of data analysed. When a much wider statistics is available, it will be possible to study how the plasma dynamics does influence and produce the statistics registered here, which is the real physics challenge of this kind of study. Anyway, the results obtained here completely agree with Tam et al. [2005], who performed intermittency analyses on the electric field data obtained by the SIERRA sounding rocket in the auroral zone. They obtained that the electric field fluctuations are more intermittent at smaller scales.

In addition, the authors think that these results, obtained on a very limited collection of data, however, point towards the convenience of systematically applying MSSA to high rate in situ data collected by satellites through the whole ionosphere. Collecting a serious "zoology" of similar ionospheric "details", and dressing them with the plasma physics motivation, will be an unprecedented progress in near-Earth space science.

Acknowledgements. This work is based on observations with the electric field instrument ICE embarked on the satellite DEMETER launched by CNES (Centre National d'Etudes Spatiales). The author thanks J.J. Berthelier the PI of this instrument for the use of the data, and CDPP (Centre des Données de la Physique des Plasmas] for the provision of these data. Space weather data from the DMSP satellites are kindly provided by the Space Vehicles Directorate of the Air Force Research Laboratory. M. Piersanti, G. Consolini, P. Diego and I. Bertello thank the Italian Space Agency (ASI) for the financial support under the contract ASI "LIMADOU scienza". 


\section{REFERENCES}

Aikio, A. T., T. Pitkänen, A. Kozlovsky, and O. Amm (2006). Method to locate the polar cap boundary in the nightside ionosphere and application to a substorm event, Ann. Geophysicae, 24, 1905-1917, doi:10.5194/angeo-241905-2006.

Akasofu, S.-I. (1966), The auroral oval, the auroral substorm and their relations with the internal structure of the magnetosphere, Planet. Space Sci., 14, 587.

Borovsky, J. E. (1993), Auroral arc thicknesses as predicted by various theories, J. Geophys. Res., 98, 6101.

Badoni D., R. Ammendola, I.Bertello, P.Cipollone, L.Conti, C.De Santis, P.Diego, G.Masciantonio, P.Picozza, R.Sparvoli, P.Ubertini, G. Vannaroni

(2018). A high-performance electric field detector for space missions, Planetary and Space Science, 153, 107119, doi: 10.1016/j.pss.2018.01.013.

J.J.Berthelier, M.Godefroy, F.Leblanc, M.Malingre, M.Menvielle, D.Lagoutte, J.Y.Brochot, F.Colin, F.Elie, C.Legendre, P.Zamora, D.Benoist, Y.Chapuis, J.Artru, R. Pfaff

(2006), ICE, the electric field experiment on DEMETER, Planetary and Space Science, 54, 5, 456-471, doi: 10.1016/j.pss.2005.10.016.

Chen F.F. (1977), "Introduction to Plasma Physics", Plenum Press, New York.

Chen, F. F. J. D Evans and D.Arnush (2002), A floating potential method for measuring ion density, Physics of Plasmas, 9, 1449; doi: 10.1063/1.1462630.

Consolini G., T. Alberti, E.Yordanova, M.F. Marcucci and M. Echim

(2017). A Hilbert-Huang transform approach to space plasma turbulence at kinetic scales, J. Phys. Conf. Ser., 900, 012003, doi:10.1088/1742-6596/900/1/012003

Diego P., I. Bertello, M. Candidi, A. Mura, G. Vannaroni, D. Badoni (2017). Plasma and fields evaluation at the Chinese Seismo-Electromagnetic Satellite for Electric Field Detector measurements, IEEE, 5, 1, doi:10.1109/ACCESS.2017.2674019

Feldstein, Y. I., S. I. Isaev, and A. L. Lebedinsky (1969), Phenomenology and morphology of aurorae, Ann. Int. Quiet Sun Year, 4, 311.

Falkovich, G. (2008). Introduction to turbulence theory, in Cardy J., Falkovich, G., and Gawedzki, K. (Eds.), Non-Equilibrium Statitical Mechanics and Turbulence, Cambridge, UK: Cambridge Univ. Press, New York.

Farge, M., M. Holschneider, and J. F. Colonna (1990), Wavelet analysis of coherent structures in two dimensional turbulent flows, in Topological Fluid Mechanics, edited by H. K. Moffat, 765, Cambridge Univ. Press, New York.
Feldstein, Y. I. (1963), Some problems concerning the morphology of auroras and magnetic disturbances at high latitudes, Geomagn. Aeronomy, 3, 183-195, 1963.

Frey, H. U. (2007), Localized aurora beyond the auroral oval, Rev. Geophys., 45, RG1003, doi:10.1029/2005RG000174.

Frisch, U. (1995), Turbulence, the Legacy of A. N. Kolmogorov, Cambridge University Press: Cambridge, UK.

Hunsucker, R. D., \&t Hargreaves, J. K. (2007). The highlatitude ionosphere and its effects on radio propagation. Cambridge University Press.

Iijima, T. and Potemra, T. (1978), The amplitude distribution of field aligned currents associated with substorms, J. Geophys. Res., 83, 599.

Kauristie, K., J. Weygand, T. I. Pulkkinen, J.S. Murphree, and P. T. Newell (1999). Size of the auroral oval: UV ovals and precipitation boundaries compared, J. Geophys. Res.: Space Phys., 104, 2321-2331.

Khorosheva, O. V. (1967). Spatial-temporal distribution of aurorae, in Aurorae, No. 16, 1-84, Academy of Sciences, Moscow.

Lagoutte D., et al. (2005), DEMETER Microsatellite Scientific Mission Center - Data User Guide, Ref: DMTOP-7-CS-6124-LPC, Rev: 1.0.

Lebreton, J.-P., S.Stverak P.Travnicek, M.Maksimovic, D.Klinge, S.Merikallio D.Lagoutte B.Poirier P.-L.Blelly, Z.Kozacek, M.Salaquarda (2006). The ISL Langmuir probe experiment processing onboard DEMETER: Scientific objectives, description and first results, Plan. Space Sci., 54, 5, 472-486; doi: 10.1016/j.pss.2005.10.017.

Moen, J., M. Lockwood, K. Oksavik, H. C. Carlson, W. F. Denig, A. P. van Eyken and I. W. McCrea (2004). The dynamics and relationship of precipitation, temperature and convection boundaries in the dayside auroral ionosphere, Ann. Geophysicae, 22, 1973-1987; doi:10.5194/angeo-22-1973-2004.

Newell, P. T., T. Sotirelis, and S. Wing (2009), Diffuse, monoenergetic, and broadband aurora: The global precipitation budget, J. Geophys. Res., 114, A09207, doi: 10.1029/2009JA014326.

Østgaard, N., S.B.Mende H.U.Frey J.B.Sigwarth, A.Åsnes J.M.Weygand, (2007). Auroral conjugacy stud-' ies based on global imaging, J. Atmos. Sol.-Terr. Phys., 69, 249.

Piersanti, M., and U. Villante (2016). On the discrimination between magnetospheric and ionospheric contributions on the ground manifestation of sudden impulses, J. Geophys. Res. Space Physics, 121, 6674-6691; doi: 10.1002/2015JA021666.

Piersanti M., T. Alberti, A. Bemporad, F. Berrilli, R. Bruno, V. Capparelli, V. Carbone, C. Cesaroni, G. Consolini, 
A. Cristaldi, A. Del Corpo, D. Del Moro, S. Di Matteo, I. Ermolli,

S. Fineschi, F. Giannattasio, F. Giorgi, L. Giovannelli, S. L. Guglielmino, M. Laurenza, F. Lepreti, M. F. Marcucci, M. Martucci, M. Mergè, M. Pezzopane, E. Pietropaolo, P. Romano, R. Sparvoli, L. Spogli, M. Stangalini, A. Vecchio, M. Vellante, U. Villante, F. Zuccarello, B. Heilig, J. Reda J. Lichtenberger, (2017). Comprehensive Analysis of the Geoeffective Solar Event of 21 June 2015: Effects on the Magnetosphere, Plasmasphere, and Ionosphere Systems, Sol. Phys., doi: 10.1007/s11207-017-1186-0.

Piersanti M., M. Materassi, A. Cicone, l. Spogli, H. Zhou and R.G. Ezquer (2018). Adaptive Local Iterative Filtering: a promising technique for the analysis of non-stationary signals, J. Geophys. Res. - Space Physics, 123, 1, 1031-1046 ; doi: 10.1002/2017JA024153.

Sojka, J. J., R. W. Schunk and W. J. Raitt (1982). Seasonal variations of the high-latitude F region for strong convection, J. Geophys. Res., 87(A1), 187-198; doi:10.1029/JA087iA01p00187.

Tam, S. W. Y., T. Chang, P. M. Kintner, and E. Klatt (2005). Intermittency analyses on the SIERRA measurements of the electric field fluctuations in the auroral zone, Geophys. Res. Lett., 32, L05109, doi:10.1029/2004GL021445.

Vellante, M., M. Piersanti, M., E. Pietropaolo (2014a). Comparison of equatorial plasma mass densities deduced from field line resonances observed at ground for dipole and IGRF models. J. Geophys. Res. 119(4), 2623. 2013JA019568. doi: 10.1002/2013JA019568

Vellante, M., M. Piersanti, B. Heilig, J. Reda, A. Del Corpo (2014b). Magnetospheric plasma density inferred from field line resonances: effects of using different magnetic field models. 2014 XXXIth URSI General Assembly Scien. Symp., 1. doi: 10.1109/URSIGASS.2014.6929941.

Villante U., M. Piersanti, Sudden impulses at geosynchronous orbit and at ground, (2011). J. Atm. Sol. Terr. Phys., 73, 1, Pages 61-76, ISSN 1364-6826, https://doi.org/10.1016/j.jastp.2010.01.008.

Wang, H., H. Lühr and S. Y. Ma (2005). Solar zenith angle and merging electric field control of field-aligned currents: A statistical study of the Southern Hemisphere, J. Geophys. Res., 110, A03306, doi:10.1029/2004JA010530.

Whitteker, J. H., G. G. Shepherd, C. D. Anger, a J. R. Burrows, D. D. Wallis, D. M. Klumpar and J. K. Walker (1978). The winter polar ionosphere, J. Geophys. Res., 83(A4), 1503-1518, doi:10.1029/JA083iA04p01503.

Xiong, C., H. Lühr, H. Wang and M. G. Johnsen (2014a). Determining the boundaries of the auroral oval from CHAMP field-aligned current signatures - Part 1, Ann. Geophysicae, 32, 609-622, doi:10.5194/angeo-32-6092014.
Xiong, C., H. Lühr, H. Wang and M. G. Johnsen (2014b), Determining the boundaries of the auroral oval from CHAMP field-aligned current signatures - Part 2, Ann. Geophysicae, 32, 622, doi:10.5194/angeo-32-622-2014.
"CORRESPONDING AUTHOR: Massimo MATERASSI Istituto dei Sistemi Complessi, Consiglio Nazionale delle Ricerche,

Firenze, Italy;

email: massimo.materassi@isc.cnr.it

(C) 2019 the Istituto Nazionale di Geofisica e Vulcanologia. All rights reserved 